Meeting Report

\title{
Symmetry, Probabiliy, Entropy: Synopsis of the Lecture at MAXENT $2014{ }^{\dagger}$
}

\section{Misha Gromov}

Institut Hautes Études Scientifiques, 35, Route de Chartres, F-91440 Bures-sur-Yvette, France; E-Mail: gromov@ihes.fr; Tel.:+33-01-6092-6600

$\dagger$ This paper was presented in MaxEnt 2014, Amboise, France, 21-26 September 2014.

Received: 2 February 2015 / Accepted: 10 March 2015 / Published: 13 March 2015

\begin{abstract}
In this discussion, we indicate possibilities for (homological and non-homological) linearization of basic notions of the probability theory and also for replacing the real numbers as values of probabilities by objects of suitable combinatorial categories.
\end{abstract}

Keywords: entropy; Bernoulli approximation; homology measures

The success of the probability theory decisively, albeit often invisibly, depends on symmetries of systems this theory applies to. For instance:

- The symmetry group of a single round of gambling with three dice has order $288=6 \times 6 \times 8$ : it is a semidirect product of the permutation group $S_{3}$ of order 6 and the symmetry group of the $3 d$ cube, that is, in turn, is a semidirect product of $S_{3}$ and $\{ \pm 1\}^{3}$.

- The Bernoulli spaces $\left(\mathbf{\square}_{p}, \boldsymbol{\vee}_{1-p}\right)^{\mathbb{Z}}, 0<p<1$, of $(\mathbf{\square}, \bullet)$-sequences indexed by integers $z \in \mathbb{Z}=$ $\{\cdots,-2,-1,0,1,2, \cdots\}$ are acted upon by a semidirect product of the infinite permutation group

$$
S_{\infty=\mathbb{Z}} \supset \mathbb{Z}=\{\cdots,-2,-1,0,1,2, \cdots\}
$$

and the (compact) group $\{ \pm 1\}^{\mathbb{Z}}=\{\boldsymbol{\square} \leftrightarrow\}^{\mathbb{Z}}$, with the role of the latter being essential even for $p \neq \frac{1}{2}$ where the probability measure is not preserved.

- The system of identical point-particles $\bullet_{i}$ in the Euclidean 3-space $\mathbb{R}^{3}$, that are indexed by a countable set $I \ni i$, is acted upon by the isometry group of $\mathbb{R}^{3}$ times the infinite permutation group $S_{\infty=I}$. 
- Buffon's probabilistic needle formula for $\pi=3.141592653589793 \cdots$ relies on the invariance of the Haar measure on the circle.

I. What happens if the symmetry is enhanced, e.g., from the permutation group $S_{\infty=I}$ to the group $G L_{\mathbb{F}}(\infty)$ of liner transformations of the vector space $\mathbb{F}^{I}$ (formally) spanned by symbols $[i], i \in I$, regarded as (linearly independent) vectors over a filed $\mathbb{F}$ ?

II. What could you do if your system is inherently heterogeneous, such as a folding polypeptide chain or a natural language, for instance?

Hilbertisation/unitarisation/quantization of set categories brought along a development of several magnificent non-commutative probability theories, e.g., of those under the headings of von-Neumann algebras, von Neumann entropy [1,2], free probabilities [3].

By comparison, the achievements of the non-unitary linearisation of probability theory are modest-just a few amusing observations.

Example 1. Linearized Loomis-Whitney-Shannon-Shearer Submultiplicativity Inequality [4,5].

Let $\Phi=\Phi\left(x_{1}, x_{2}, x_{3}, x_{4}\right)$ be a 4-linear function (form) over some field (where the variables $x_{i}$ run over some vector spaces $\left.X_{i}\right)$. Then the ranks of the following four bilinear forms $\Phi\left(x_{1}, x_{2} \otimes x_{3} \otimes x_{4}\right), \Phi\left(x_{1} \otimes\right.$ $\left.x_{2}, x_{3} \otimes x_{4}\right), \Phi\left(x_{1} \otimes x_{3}, x_{2} \otimes x_{4}\right)$ and $\Phi\left(x_{1} \otimes x_{4}, x_{2} \otimes x_{3}\right)$ satisfy

$$
(\operatorname{rank}[1,234])^{2} \leq \operatorname{rank}[12,34] \cdot \operatorname{rank}[13,24] \cdot \operatorname{rank}[14,23] .
$$

Example 2. Homology Measures [6].

Homologies $H_{*}(X)=\oplus_{i} H_{i}(X)$ of topological spaces $X$ and natural subgroups in $H_{\star}$ are graded Abelian groups: their ranks are properly represented not by individual numbers $r_{i}$, but by Poincaré polynomials $P_{X}(t)=\sum_{i} r_{i} \cdot t^{i}$.

The polynomial valued set function $U \mapsto P_{U}, U \subset X$, has some measurelentropy-like properties that become more pronounced for the ideal valued function that assigns the kernels

$$
\operatorname{Ker}_{X \backslash U} \subset H^{*}(X ; A)
$$

of the inclusion/restriction cohomology homomorphisms for the complements $X \backslash U \subset X$ for subsets $U \subset X$,

$$
U \mapsto \mu^{*}(U)=_{\text {def }} \operatorname{Ker}_{X \backslash U}=_{\text {def }} \operatorname{Ker}\left[H^{*}(X ; A) \rightarrow H^{*}(X \backslash U ; A)\right],
$$

for some Abelian (cohomology coefficient) group A.

The basic properties of this $\mu^{*}$ (stated slightly differently in topology textbooks) have an attractive measure theoretic flavour. Namely,

$\mu^{\star}(U)$ is additive for the sum-of-subsets in the group $H^{\star}(X ; A)$ and, if $A$ is a commutative ring, then $\mu^{*}$ is super-multiplicative for the the - -product of ideals:

$$
\mu^{*}\left(U_{1} \cup U_{2}\right)=\mu^{*}\left(U_{i}\right)+\mu^{*}\left(U_{2}\right)
$$


for disjoint open subsets $U_{1}$ and $U_{2}$ in $A$, and

$$
\mu^{*}\left(U_{1} \cap U_{2}\right) \supset \mu^{*}\left(U_{1}\right) \smile \mu^{*}\left(U_{2}\right)
$$

for all open $U_{1}, U_{2} \subset A$.

Next, given a linear subspace $\Theta \subset H^{*}(X ; A)$, let

$$
\mu_{\Theta}(U)=\Theta \cap K e r_{X \backslash U}
$$

and, assuming $A$ is (the additive group of) a field, denote the rank of $\mu_{\Theta}(U)$ over this field by $\left|\mu_{\Theta}(U)\right|=$ $\left|\mu_{\Theta}(U)\right|_{A}$.

Linearized Matsumoto-Tokushige Separation Inequality in the N-torus.

Let $U_{1}, U_{2} \subset \mathbb{T}^{N}$ be non-intersecting (closed or open) subsets and let

$$
\Theta_{1}=H^{n_{1}}\left(\mathbb{T}^{N} ; A\right), \text { and } \Theta_{2}=H^{n_{2}}\left(\mathbb{T}^{N} ; A\right)
$$

for $n_{i} \leq N / 2, i=1,2$, and some field $A$. Then

$$
\left|\mu_{\Theta_{1}}\left(U_{1}\right)\right| \cdot\left|\mu_{\Theta_{2}}\left(U_{2}\right)\right| \leq c \cdot\left|\Theta_{1}\right| \cdot\left|\Theta_{2}\right|
$$

for $c=n_{1} n_{2} / N^{2}$ and where, observe, $\left|\Theta_{i}=\wedge^{n_{i}} A\right|=\left(\begin{array}{l}N \\ n_{i}\end{array}\right)$.

If we think of the torus $\mathbb{T}^{N}$ as a physical system of $N$ uncoupled linear oscillators then the "measures" $\mu^{*}(U)$ and/or $\mu_{\Theta}(U)$ may be interpreted as

"the numbers of persistent degrees of freedom" of this system that are observable from $U$.

Probabilistic/entropic interpretation of homology, which is kind of "dual" to "homological interpretation of entropy-like invariants" by Bennequin [7], and also by Drummond-Cole et al. [8,9], is also possible for "coupled systems" [10] where particularly attractive ones are systems of moving disjoint balls in space where the configuration spaces of these systems support rich homology structures that are induced from the classifying spaces of (subgroups of) infinite symmetric groups $S_{\infty=I}$ [11], that is expanded/corrected in [12].

A mathematical study of "loose structures" such as what you find in biology and linguistics needs generalisations that would allow a use of relaxed, rather than enhanced, symmetries.

For instance, just to warm up, one may start by elaborating on the category theoretic definition of the entropy suggested "In a Search for a Structure, Part 1: On Entropy" [13], where the entropy of a finite probability space $P=\left\{p_{i}\right\}, p_{i}>0, \sum_{i} p_{i}=1$, comes as the class $[P]_{\text {Gro }}$ of $P$ in the Grothendieck group $\operatorname{Gro}(\mathcal{P})$ of the topological category $\mathcal{P}$ of finite probability spaces $P$ and probability/measure preserving maps $P \rightarrow Q$ with a properly defined topological structure in $\mathcal{P}$.

Since the group $\operatorname{Gro}(\mathcal{P})$ is isomorphic to the multiplicative group of positive real numbers [13] - this is a reformulation of the Bernoulli law of large numbers - the Grothendieck class $[P]_{\text {Gro }}$ can be identified with $\exp \operatorname{ent}(P)$.

In general, such a Grothendieck-style entropy would be not a number valued function of any kind, but (not quite) a functor from an elaborate combinatorial (not quite) category, e.g., comprised of fragments 
of a natural language with some (not always composable) "morphisms/arrows" between them, to some "simple category" e.g., the category of weighted trees.

The so modified probability/entropy theory is badly needed for designing algorithms that would model what we call (ego)learning described in "Ergostructures, Ergodic and the Universal Learning Problem" [14] and in "Understanding Languages and Making Dictionaries" [15], (in preparation) but I have not progressed much in pursuing this direction yet.

\section{Acknowledgments}

I want to thank Frederic Barbaresco for his interest in the subject matter of this paper and for inviting me to the MaxEnt'14 meeting in Amboise, France, and the anonymous referees for their friendly comments and suggestions.

\section{Conflicts of Interest}

The author declares no conflict of interest.

\section{References and Notes}

1. Parthasarathy, K.R. An Introduction to Quantum Stochastic Calculus; Modern Birkhäuser Classics; Springer: Basel, Switzerland, 1992.

2. Meyer, P.-A. Quantum Probability for Probabilists, 2nd ed.; Lecture Notes in Mathematics; Springer: Berlin/Heidelberg, Germany, 1995.

3. Nica, A.; Speicher, R.; Voiculescu, D. Free Probability Theory. Available online: https://www.birs. ca/workshops/2004/04w5028/report04w5028.pdf (accessed on 10 March 2015).

4. Gromov, M. Entropy and isoperimetry for linear and non-linear group actions. Groups Geom. Dyn. 2008, 2, 499-593. Available online: http://www.ihes.fr/ gromov/topics/grig-final-june11-08.pdf (accessed on 10 March 2015).

5. Gromov, M. Six Lectures on Probabiliy, Symmetry, Linearity. Available online: http://www.ihes. fr/ gromov/PDF/probability-paris-Oct-2014.pdf (accessed on 10 March 2015).

6. Gromov, M. Singularities, expanders and topology of maps. Part 2: From combinatorics to topology via algebraic isoperimetry. Geom. Funct. Anal. 2010, 20, 416-526. Available online: http://www.ihes.fr/ gromov/PDF/morse2_gafa.pdf (accessed on 10 March 2015).

7. Bennequin, D. Homological interpretation of entropy-like invariants. In Proceedings of 34th International Workshop on Bayesian Inference and Maximum Entropy Methods in Science and Engineering, 21-26 September 2014, Amboise, France.

8. Drummond-Cole, G.C.; Park, J.-S.; Terilla, J. Homotopy Probability Theory I. J. Homotopy Relat. Struct. 2013, doi: 10.1007/s40062-013-0067-y.

9. Drummond-Cole, G.C.; Park, J.-S.; Terilla, J. Homotopy Probability Theory II. J. Homotopy Relat. Struct. 2013, doi: 10.1007/s40062-014-0078-3.

10. Bertelson, M.; Gromov, M. Dynamical Morse Entropy; Modern dynamical systems and applications; Springer: Berlin, Germany, 2010. Available online: www.ihes.fr/ gromov/PDF/ dynamical-entropy-final-bis.pdf (accessed on 10 March 2015). 
11. Gromov, M. Number of Questions. Available online: http://www.ihes.fr/ gromov/PDF/ sept2014-copy.pdf (accessed on 10 March 2015); Section 4, pp. 82-102.

12. Gromov, M. Morse Spectra, Homology Measures and Parametric Packing Problems. 2015, in preparation.

13. Gromov, M. In a Search for a Structure, Part 1: On Entropy. Available online: http://www.ihes.fr/ $\sim$ gromov/PDF/structre-serch-entropy-july5-2012.pdf (accessed on 10 March 2015).

14. Gromov, M. Ergostuctures, Ergologic and the Universal Learning Problem: Chapters 1,2. Available online: http://www.ihes.fr/ gromov/PDF/ergologic3(1).pdf (accessed on 10 March 2015).

15. Gromov, M. Understanding Languages and Making Dictionaries. 2015, in preparation.

(C) 2015 by the author; licensee MDPI, Basel, Switzerland. This article is an open access article distributed under the terms and conditions of the Creative Commons Attribution license (http://creativecommons.org/licenses/by/4.0/). 